\title{
Le Paradoxe de la Claustration dans Rose et Blanche de J. Sand
}

Caroline JUMEL

$\mathrm{J}$ - Sand, l'auteur de Rose et Blanche correspond à la fusion des noms de Jules G. Sandeau et d'Aurore Dudevant à l'aube de la création de son nom de plume et la part investie par chacun des auteurs quant à l'écriture de Rose et Blanche a été l'objet de nombreuses controverses. Il ne s'agit pas ici de déterminer s'il existe un auteur dominant ou non, mais plutôt de souligner que George Sand connaissait les lieux qui sont décrits dans l'œuvre et qu'il existe ainsi une ressemblance frappante avec certaines personnes qu'elle avait côtoyées.' En outre, de nombreux aspects et thèmes de cette écriture reflètent ce qu'elle développera au cours des années qui suivront, notamment, les thèmes de l'orpheline, de la claustration et du mariage en relation avec le thème de l'espace. J'analyserai donc la représentation du couvent selon G. Sand, comme espace féminin complexe et paradoxal. En effet, le couvent sert à la fois de refuge contre l'oppression mais aussi de structure carcérale. Le couvent est donc représenté comme un refuge problématique. En cela, l'écriture de G. Sand annonce l'analyse féministe de l'espace social.

J'organiserai cette étude en deux parties distinctes, liées à l'espace dominant de l'œuvre: le couvent, et ses fonctions dans le roman. Dans la première partie, je montrerai que le couvent fonctionne d'abord pour les deux protagonistes féminines comme un refuge face à l'oppression. Puis, 
dans la seconde partie, j'étudierai la fonction du couvent en tant qu'espace carcéral.

Le roman débute sur la rencontre des deux jeunes filles dont les destinées paraissent être diamétralement opposées. Rose est une petite comédienne de province, manipulée par sa mère aux mœurs plus que douteuses. Rose n'a pas de père que l'on connaisse, et se trouve être totalement livrée aux désirs de sa mère qui voit en Rose, ou plutôt, en son corps, une source éventuelle de revenus. Rose se révolte contre le fait que sa mère veuille faire d'elle un objet de prostitution mais ne voit pas d'issue à son problème. L'éducation qu'elle a reçue consiste à se soumettre aux volontés de sa mère. La Primerose (la mère de Rose) utilise sans scrupule la tendresse maternelle pour pousser sa fille à la prostitution: " Voyons, Rose [. . ] il est temps de faire quelque chose pour ta mère [. . .] crois-tu qu'il n'y ait pas quelque mérite à t'avoir gardée avec moi? [...] Alors ma mignonne, récompense donc ma tendresse maternelle » (69). La mère souligne même le fait qu'elle a eu du mérite de ne pas avoir abandonné Rose, ce qui prouve qu'elle y a donc pensé. Rose est véritablement vertueuse de ne pas avoir sombré dans le vice plus tôt (elle a alors 15 ans) et de continuer à refuser cette solution pour améliorer les revenus du couple mère-fille. Rose critique l'attitude de sa mère et ne conçoit pas une relation avec un homme sans amour: « Tromper, mentir, être vendue, payée, non, jamais! » (71). Cependant la Primerose, après avoir évoqué la tendresse maternelle, utilise la dernière arme qu'elle pense de poids pour faire fléchir sa fille: la religion: « Allez, on voit bien que vous ne connaissez pas les commandements de Dieu ... Tes père et mère honoreras ... » (71). Il est remarquable que Rose refuse d'être la complice de sa mère, vu que toute son éducation lui vient de cette dernière. Mais la résistance de Rose se heurte à la triste réalité de sa propre condition sociale. Elle reconnaît n'avoir rien d'autre que sa mère. De crainte de tout perdre (sa mère et la vie qu'elle mène) car aucune solution ne se profile pour elle à l'horizon, elle finit avec dégoût par accepter le marché conclu par sa mère avec Laorens; sa vente en tant qu'objet de plaisir sexuel. Il ressort clairement que si Rose cède, c'est qu'elle n'a pas d'alternative. Hors du monde thêatral, elle ne voit pas ce qu'elle pourrait faire. Étant orpheline du père, elle n'a pas d'autre éducation que celle donnée par sa mère, donc basée sur la vie au théâtre. L'ignorance et l'éducation limitée qu'elle a reçue s'avèrent être un obstacle au désir de vertu de Rose, ainsi que la misère de sa condition sociale. La pauvreté ne peut offrir aux jeunes filles le moyen de s'échapper de cet espace dégradé et dégradant. Rose se trouve dans un cercle vicieux (au propre et au figuré). Pour subsister dans ce 
monde qui n'a rien à offrir aux jeunes filles pauvres, il faut continuer dans la seule voie ouverte par sa mère. Le statut d'orpheline de Rose, renforce l'idée que sans homme pour subvenir à ses besoins, la femme doit se débrouiller seule et les moyens d'obtenir l'indépendance sont limités précisément à ceux que Rose refuse.

L'auteur représente la femme en tant que victime et les mères, ou les tutrices, participent à perpétrer cette condition de victime, comme le souligne Rose: "Partout les vieilles sont toujours mauvaises pour les jeunes » (15). Un événement décisif se produit en faveur de Rose. Horace, l'ami de Laorens qui faisait partie du marché conclu avec la Primerose, prend la place de son ami qui est trop saoul pour aller retrouver Rose. Horace voyant Rose victime de sa condition, se révolte et lui propose d'en finir avec cette vie et avec sa mère: « Tu aurais servi de philtre entre les mains d'une sorcière pour ranimer les sens éteints de quelque vieillard usé!...Abandonnée, maudite, rongée de maux infâmes, tu aurais traîné dans la boue des rues, souri aux outrages des passants, et tu serais morte dans une léproserie [. . . Providence [. . .] qu'as-tu fait pour les pauvres filles? » (90). Horace décrit exactement ce que serait devenue Rose si elle avait continué dans la voie dans laquelle sa mère la poussait et il fait ici le portrait de toute une catégorie de femmes, poussée par la misère à se vendre d'une manière ou d'une autre. Cependant, le côté philanthrope et altruiste d'Horace envers Rose (lorsqu'il décide de la faire entrer au couvent) n'est pas véritablement sa propre nature. Il prend la décision d'aider Rose car un secret peu à son honneur sur son passé le ronge et il désire se racheter d'une manière ou d'une autre. Il est coupable d'avoir violé une jeune fille, Denise. A l'époque où elle est introduite au lecteur, elle se nomme Denise et est «idiote ». Denise est ensuite confiée à Horace, qui la fait envoyer au couvent, après la mort de son père. Blanche (Denise) au couvent retrouve ses capacités intellectuelles mais ne recouvre pas la mémoire de son passé. Le lecteur apprend au cours de la diégèse que Denise (j'utilise le nom Denise pour parler de Blanche hors du couvent, et à l'époque où elle était « idiote ») a été violée par Horace, alors ami du père de celle-ci. Le parallèle établi ici, entre les deux héroïnes principales, est donc qu'hors du couvent, elles vivaient dans une misère relative et respective à chacune, et qu'également elles ont été victimes.

Le problème de la transmission d'éducation et de situation sociale de mère en fille est d'ailleurs clairement exprimé dans la situation même de la Primerose. Cette dernière n'est pas coupable de vouloir transmettre son héritage socioculturel à sa fille. Au contraire, elle croit bien faire car elle a reçu de sa propre mère ce même genre d'éducation: « Et cette femme, si 
misérablement dépravée par l'éducation que lui avait donnée sa mère, et qu'elle transmettait à sa fille comme un héritage qu'elle devait garder avec orgueil, cette femme qui portait si haut la religion de ses principes et la dévotion du vice... » (93). La Primerose n'est pas non plus coupable mais bien victime de la société et de son manque de moyens à l'égard des filles. G. Sand met le doigt ici sur un point sensible qui sera de plus en plus d'actualité au cours du siècle: l'éducation des jeunes filles de milieux sociaux défavorisés. Françoise Mayeur souligne cette absence totale de désir de changement en ce qui concerne ces jeunes filles :

A la séparation selon les sexes, s'ajoute une discrimination sociale. Au XIXème siècle, les filles des milieux aisés ne sont pas en général élèves des établissements destinés aux classes populaires [. . .] Cette ségrégation en introduit une autre : à l'époque, le sentiment est encore très fort qu'il faut dispenser à l'enfant une instruction et une éducation en harmonie avec la position sociale qu'il occupera probablement, d'après son origine. Personne n'a l'idée et surtout pas pour les filles, que l'éducation, précisément, pourrait donner à l'enfant une position supérieure à celle de ses parents. (8-9)

G. Sand représente clairement cette vision de son siècle, lorsque Rose est introduite au couvent comme Mademoiselle de Beaumont. Sans cette mascarade et cette assimilation à la bourgeoisie, elle n'aurait pas été admise dans ce couvent pour filles de riches. Lorsqu'elle est démasquée, les commentaires qui suivent soulignent bien le fait que les différentes classes sociales ne doivent pas être mélangées. Blanche, tout comme Rose, vient d'un milieu social défavorisé. Elle n'a plus de mère, et son père vit de pêche sur l'estuaire de la Gironde. Cependant, avant la mort de Lazare (le père de Denise), la vie de Denise est représentée comme étant paisible et heureuse. G. Sand la situe dans un contexte bucolique, baignant ses pieds dans l'eau, ou encore, à l'avant du bateau de son père, appréciant les moments simples offerts par la nature. La nature est en contraste avec la société, la culture. Elle offre un refuge à la femme. ${ }^{2}$ Elle permet notamment d'échapper aux normes répressives de la société/culture. La nature représente un espace extérieur non-social que l'on pourrait également qualifier d'intérieur subjectif, ou d'espace de subjectivité. Hors de la réalité, car Denise vit dans un monde bien à elle, la femme peut être heureuse. Mais une fois en contact avec cette même réalité, une série de déboires et de traumatismes s'ensuit. La fonction attribuée à l'«idiote » contraste avec celle de Blanche, revenue à ses sens et ses capacités intellectuelles. Elle souligne le bonheur lié à l'insouciance, à la naïveté et 
à la méconnaissance du monde social tel qu'il se présente réellement pour les femmes. Janet Beizer, à ce titre, établit l'existence d'une part de sublime dans cette sorte d'abrutissement: « Speech loss opens the space of an incommunicable sublime » (54). Denise ne parle jamais, ou du moins, le lecteur ne l'entend jamais prononcer la moindre parole venant de sa propre initiative. Elle ne fait que répéter ce qu'elle entend. Ce mutisme oral — car Denise n'est pas réellement muette - révèle donc bien cet espace subliminal qu'elle habite, et que peut-être, inconsciemment, elle ne désire pas quitter, comme la suite le prouve : «Il semblait que cette jeune fille n'appartint pas à la même sphère, n'eût point part à la vie des autres créatures » (182). L'ignorance et l'insouciance de Denise font d'elle un être à part, différent des femmes confrontées à la réalité. G. Sand la compare à une nymphe et la divinise: « Avec sa grande taille, son coloris brillant, son attitude ferme et calme, un pied sur la joue de la chaloupe et les bras croisés sur son sein, elle était belle et mâle comme une divinité sauvage : c'était une nymphe des écueils de l'Océan travestie en fille de marinier " (186). Denise n'appartient pas au domaine des simples mortels mais des dieux, dans cette description.

Il convient de souligner que ce portrait l'élève dans une sphère supérieure et intouchable dans laquelle le bonheur existe. L'extérieur du couvent, et plus particulièrement la nature associée dans le cas de Blanche à une certaine béatitude, due à son ignorance, contraste avec le recouvrement de sa mémoire et de ses capacités intellectuelles dans l'espace social religieux. Cette aphasie se retrouve chez de nombreux personnages féminins de G. Sand. Elle permet de créer une sorte de bouclier protecteur face à la société et ses réalités. Une fois en contact avec la réalité, Blanche est sceptique quant à un éventuel bonheur, tout comme Rose.

L'espace social qu'est l'extérieur du couvent, au début de l'histoire, montre donc deux jeunes filles pauvres et orphelines d'un parent, et également traumatisées par leur environnement. Rose ne voit pas poindre de perspective de bonheur sur son horizon social tant qu'elle subit l'influence de sa mère, dans la sphère du spectacle. Denise était heureuse, car ignorante, mais il ressort néanmoins par son viol qu'elle a été victime. Les deux jeunes filles, Rose et Blanche, ont donc ce statut de victime dans la première partie de leur vie, qui compose également la première partie de cette étude. La société n'a rien à leur offrir, sinon des souffrances, ainsi que des humiliations physiques et morales.

Le couvent vers lequel les deux protagonistes sont dirigées - car il faut avant tout souligner que la société les pousse à entrer au couvent- 
est représenté au premier abord comme un asile; asile effrayant pour Rose, asile désiré pour Blanche. La vision du couvent selon Rose est celle de l'austérité et de l'ennui. Mais elle est consciente du fait que sa situation sociale ne lui offre pas vraiment d'autre choix: « On m'a souvent dit, qu'il n'y avait pas de choix pour une orpheline (et c'est ainsi que je me considère) entre la défaveur publique et la retraite absolue. Ma mère me menaçait du couvent... " (115). L'idée que Rose se fait du couvent est également due aux descriptions qu'en a fait sa mère. En l'utilisant comme une menace, elle l'a fait paraître comme quelque chose de terrible et de terrifiant. De plus, au cours de la période de transition entre sa vie d'actrice et sa vie au couvent, Rose se retrouve chez mademoiselle Cazalès, la sœur d'Horace, qui la recueille avant de la faire entrer au couvent. Là, elle y rencontre de nombreux dévots qui lui font paraître le monde religieux sous un angle peu attrayant et peu flatteur. On s'y ennuie et surtout, l'hypocrisie y règne: «Ce que je vois de cette société là, dit Rose [...] me déplaît tant, que s'il n'y avait pas de couvents dans le monde, je crois que je retournerais au théâtre; ici on est vil avec plus de décence » (146). Le couvent apparait comme l'ultime et unique solution après avoir considéré le théâtre et la société. En effet, pour Rose, le couvent ou le théâtre paraissent composer la seule alternative possible. Une fois au couvent, ses appréhensions se renforcent, et les dires de sa mère sur le couvent trouvent une justification. Le couvent est synonyme de débarras pour les jeunes filles.

Le couvent est représenté de manière ambivalente par l'attirance pour un monde calme et à l'abri du vice, et l'aspect repoussant de la claustration et de l'abrutissement lié à celle-ci, ainsi que l'intolérance de certains religieux. Cependant Rose, malgré sa peur et ses appréhensions, ne voit pas d'autre issue: "Qu'irais-je chercher dans le monde? une famille? Je n'en ai pas; un mari? Je n'ai pas de dot; une mère? » (286). Elle se sent rejetée par la société, abandonnée, et n'a plus le désir d'y retourner. Elle développe un sentiment de haine et de dégoût pour le monde extérieur au couvent. Elle décide donc de rester avec les religieuses. Mais rapidement, son état mental se dégrade et les images qui sont associées au couvent sont celles de la mort. La réclusion est décrite par Rose comme étant contre nature : «Va, la réclusion est une vertu hors nature. Elle ne sert à rien et Dieu ne peut pas nous en faire un mérite. Je voudrais sortir! » (321). L'inaction et l'ennui règnent et ne correspondent pas aux aspirations normales de jeunes filles. Rose se met à rêver de liberté et d'extérieur du couvent. Elle idéalise le monde de souffrances et d'humiliations qu'elle a quitté et s'échappe un soir du couvent pour aller respirer l'air de la liberté. 
Elle y revient après son escapade nocturne et tombe malade d'ennui. Sa mère vient finalement la sortir du couvent et Rose remonte sur les planches.

Ce changement d'espace est également associé à un changement d'identité, révélé par l'onomastique et un jeu de masques. Il convient de noter ici le parallélisme entre Rose et l'héroöne éponyme du roman Consuelo, au regard de leur place dans l'espace physique: la sortie du couvent suivie de l'accession immédiate à la scène. Rose en changeant d'espace, change aussi de nom. Elle prend le pseudonyme de « La Coronari ». Ce changement onomastique souligne également le changement d'identité selon les différents espaces et les différentes activités des héroünes (Consuelo sur scène se nomme La Porporina). L'idée du masque semble prendre sens, et $\mathrm{G}$. Sand souligne ainsi le rôle joué par la femme sur la scène publique. Elle n'est pas véritablement ellemême si elle doit s'affubler d'un pseudonyme, ou alors, elle ne parvient à être elle-même en société qu'en ayant recours à une certaine forme de mascarade. Cet argument est confirmé par la suite de la diégèse: Rose (ou plutôt la Coronari) se déguise en homme et se fait appeler Tony pour jouer un tour à Horace. Celui-ci sera dupe jusqu'à ce que « Tony » décide de lever son masque et de mettre fin à la supercherie qui a amené Horace à échanger avec Tony des pensées d'ordre philosophique. Les seuls moments où Rose ne porte ni masque ni panoplie (hors de la scène et hors du couvent), sa vie n'est faite que de tortures morales, particulièrement au contact d'Horace, son "sauveur " mais aussi bourreau. Sous les masques masculins, que ce soit sur la scène théâtrale où elle joue le rôle d'un homme ou sur la scène publique où elle se déguise en homme (Tony), Rose est brillante et admirée. Ces publics l'admirent donc pour ce qu'elle représente et non pour ce qu'elle est réellement.

En homme, Rose est traitée d'égal(e) à égal et les discussions engagées prennent un tour philosophique, alors que sous ses habits de femme, Rose ne fait que susciter des paroles liées à son physique. Ce jeu de masque souligne la dichotomie homme/femme, esprit/corps. Le travestissement prouve ici que la femme est capable d'appartenir à la sphère spirituelle. Elle désire être aimée et être reconnue en tant qu'ellemême, c'est-à-dire, venant de ce milieu défavorisé, sans éducation, mais avide de vertu et d'amour. Mais Rose sous ses véritables traits ne suscite pas ces sentiments. Au contraire, elle repousse Horace (dont elle désire être aimée) qui se laisse dévorer par les conventions sociales et les préjugés de classe. Ainsi, à nouveau, le monde extérieur est une source de souffrances et d'humiliations pour Rose.

Pour Rose, tout comme pour Denise (Blanche), l'espace social n'est que déception et surtout viol de la femme, qu'il soit physique ou moral: 
«La vie commune était un poison lent, il lui fallait une existence d'exception [.. .] Chaque jour l'expérience déflorait ses pensées et l'isolait de cette société toute d'usage et de conventions. Dès son enfance, elle s'était sentie au-dessus de la sphère où elle était destinée à tourner » (428). Le terme "déflorait » souligne bien l'association à la virginité et par conséquent au viol. La nature du viol de Rose est effectivement d'ordre moral, mais incontestablement, la société n'est qu'agression et violation de la nature intime des jeunes filles. Rose ne peut se satisfaire de cette relation artificielle et de dépendance à la société. L'alternative ne se pose plus: il ne lui reste plus que le couvent. Si sa condition ne peut lui apporter l'amour qu'elle souhaite, elle renonce alors à cette condition, qui, de toute évidence, ne satisfait pas ses aspirations naturelles. L'art véritable auquel elle se voue est bafoué, et l'amour auquel elle aspire lui est refusé. Il ne lui reste donc qu'à faire ses adieux à la scène sociale et se retirer du monde. Rose enlève donc son habit social d'actrice pour finalement " prendre l'habit ». Ironiquement, ces passages d'une identité à une autre soulignent que Rose ne peut, et ne pourra jamais, être véritablement ellemême. Il faut toujours une deuxième peau à Rose pour pouvoir évoluer dans une sphère, quelle qu'elle soit. Sa véritable identité lui est finalement refusée: «Elle fut bientôt lasse de cette vie factice qui n'apportait pas une joie à son cœur. Avide d'affection, dévorée par son âme ardente, mais trop supérieure au petit monde froid et superbe qui l'entourait, elle le traversa sans y trouver où s'attacher» (428). Rose se résout enfin à retourner au couvent puisque le monde ne répond pas à ses aspirations.

Par opposition à l'asile qu'est enfin le couvent pour Rose, G. Sand souligne le paradoxe de la fonction du couvent. S'il devient finalement un asile pour Rose, il se transforme en véritable espace carcéral pour Blanche. Effectivement, Blanche, restée au couvent lors de la sortie de Rose, se languit. Elle s'y ennuie et émet son désir d'en sortir une fois son noviciat terminé. Cependant, le directeur du couvent s'y oppose, car les religieuses se sont efforcées de faire croire à ce dernier que Blanche désirait rejoindre Rose et sa vie de dépravation. Il est intéressant de se demander quelles sont les motivations qui poussent les religieuses à vouloir empêcher Blanche de sortir. Il pourrait s'agir d'une sorte de revanche envers Rose qui a dupé tout le couvent en faisant croire qu'elle descendait de souche noble, sans quoi son acceptation au couvent aurait été rejetée. Au couvent, Rose étant la meilleure amie de Blanche, l'association est donc systématique. En punissant Blanche, les religieuses pensent punir Rose. Une autre possibilité est qu'elles essaient tout simplement de sauver Blanche de la perdition. 
Une troisième possibilité est, qu'à l'époque, des tensions opposent les partisans de la laïcité et les partisans de la religion en matière d'éducation. Blanche pourrait n'être que le bouc émissaire qui se trouve entre ces deux camps. L'église est dans ce cas, prête à tout pour récupérer le plus possible d'éléments dans son camp. Mayeur note cette dissension relative à la première moitié du XIXème siècle : «Dispensée d'abord avec ménagements, chichement en quelque sorte, dans une intention entièrement désintéressée, pour l'agrément de la société profane, l'instruction supérieure des jeunes filles s'est chargée au cours du siècle de nouvelles implications. L'Eglise y a vu un moyen de reconquête des élites, partant de la société dans son ensemble » (9). Le lecteur sait pertinemment que Blanche n'appartient nullement à l'élite sociale et il est d'ailleurs le seul à savoir, avec Horace, que Blanche vient d'une classe sociale pauvre. Elle a été acceptée au couvent car tout le système institutionnel religieux s'est fait dupé par Horace qui a présenté Blanche comme une bourgeoise. L'ecclésiastique qui la force à rester au couvent n'est pas donc au courant de cette tromperie. Blanche se trouve être tout simplement un des moyens, pour l'église, de régler ses comptes avec la société laïque qui se développe.

Quoiqu'il en soit, le résultat est le même. Blanche se retrouve séquestrée à l'intérieur du couvent, sous les apparences protectrices des supérieures. Elle finit donc par se plier aux désirs et conseils des religieuses, et décide de s'installer définitivement au couvent, sans se douter de toutes les manigances qui ont eu lieu à son insu. Le lecteur retrouve à cette période du roman, les commentaires émanant directement du narrateur : « Il eût été difficile de deviner qu'une pauvre fille, dans toute la force de l'âge, dans les premiers jours de sa beauté, allait être fiancée à une réclusion éternelle » (386). Le parallèle établi ici est indiscutablement le couvent et la prison.

À travers la voix du narrateur, G. Sand dénonce ce sacrifice et souligne le manque de liberté de choix donné à Blanche, quant à son avenir.

Cependant, un évènement inattendu va faire sortir Blanche du couvent. Un ecclésiastique persuade Blanche qu'elle est coupable et non victime du viol, et que pour expier sa faute, l'unique solution est de se marier avec Horace. La situation se trouve complètement renversée. Les valeurs sont bafouées, la logique se transforme en aberration totale et Blanche se retrouve épouse de son violeur, malgré elle. La société entière a conspiré contre Blanche (l'onomastique prend ici son sens plus que jamais, Blanche symbolise l'innocence, la pureté et la vérité) et la pousse dans un espace digne d'être assimilé à un asile psychiatrique 
sans murs. Le non-sens devient le « bon sens », l'abjection est transformée en purification, le mariage est présenté comme l'unique moyen de salut et va pourtant se révéler mortel pour Blanche. La vue d'Horace à ses pieds, le soir des noces, produit un effet d'analepse car Blanche se retrouve mentalement projetée à l'époque du viol, se voit à nouveau devenir la proie offerte publiquement à un monstre sexuel: « La malheureuse créature semblait une victime offerte en expiation d'un crime qui n'était pas le sien » (416). Son âme bouleversée ne peut en supporter plus, et elle meurt de panique et de dégoût à l'idée de devenir, une seconde fois, la victime du même homme. Cet acte de jeter Blanche dans les bras de son bourreau évoque la victime que l'on jette dans la fosse aux lions et dont les spectateurs se délectent, car ils assouvissent enfin leurs pulsions agressives. Cette catharsis délivre les religieux du poids de leur culpabilité et des peurs liées à celle-ci. Blanche est donc un objet de purification et de délivrance pour ces âmes malsaines qui l'entourent.

Pour Blanche, la seule sphère supportable possible, après bien des hésitations, était devenue le couvent. L'extérieur a été pour elle une succession de viols, physiques et moraux. Le couvent, même si l'hypocrisie et la méchanceté y régnaient, était l'unique solution et créait un semblant de bonheur. G. Sand établit un contraste entre le couvent où la présence féminine domine, et l'extérieur où Blanche vivait sous l'autorité et le regard patriarcal.

Pour ces deux jeunes filles issues de milieux pauvres, la société n'a rien d'autre à offrir en l'absence de père et de dot, sinon le couvent. Estil judicieux de définir alors le couvent comme un débarras pour jeunes filles? Il est difficile de répondre à cette question étant données les variantes qui composent l'utilisation du couvent. Pour les jeunes filles pauvres, il s'agit effectivement d'un endroit décent où les mettre et les protéger d'une société impitoyable. La preuve en est que lorsque Blanche est sans protection, elle est violée, souillée et surtout se trouve sans défense. La situation de Rose est identique. Elle était vouée à être traînée dans la boue si elle ne s'était pas tournée vers le couvent. Le couvent s'avère donc dans le cas de Rose et Blanche, être un asile. Mais s'il est un asile, il faut souligner qu'il est l'unique refuge offert à ces filles. Aucune alternative ne s'offre. L'enfermement des jeunes filles tend ainsi à réduire la dépravation humaine et l'hégémonie patriarcale se satisfait de cela, car la mise à l'écart de ces filles permet au pouvoir d'avoir moins de pauvreté et de déchéance sous les yeux. Le pouvoir enferme, afin de mieux cacher cet échec, qui cependant, lui revient. 
Après leurs déboires respectifs, le couvent devient incontestablement un refuge. La liberté mentale l'emporte finalement sur la liberté physique. Il vaut mieux pouvoir être libre de ses pensées, plutôt que d'être physiquement libre, mais sous le joug inflexible d'un monde incompréhensif et incompréhensible. La clôture du roman, parallèle à la nouvelle et ultime clôture physique de Rose, souligne cependant l'aspect libérateur et protecteur de cette claustration:

L'air de la liberté n'est plus nécessaire à celle qui a traversé le monde et connu les hommes. De l'amitié, du loisir pour étudier, du soleil, de l'air et des fleurs, c'est ce dont se compose une existence de religieuse, et que faut-il de plus au cœur que l'amour et la gloire ont trahi? Si l'on détruisait les couvents, quelques existences rejetées de la société, quelques âmes trop délicates pour le grossier bonheur de la civilisation n'auraient plus de terme moyen entre le spleen et le suicide. (433)

Le lecteur assiste donc paradoxalement à une délivrance dans l'acte d'enfermement volontaire. La femme s'émancipe en la présence de femmes. Ce réseau féminin paraît être la seule solution acceptable et surtout la seule échappatoire à l'enfermement moral de la société. L'amitié prend une valeur supérieure à celle de l'amour. Si la femme veut être heureuse, il lui faut fuir les hommes et la société qu'ils dirigent. $K$. Crecelius souligne l'aspect répressif de la société du début du dix-neuvième siècle pour les femmes et le manque de liberté accordée à ces dernières pour exprimer et vivre leurs aspirations: «The convent is seen here as it was for many centuries: the sphere in which women could realize themselves and create destinies for themselves outside of the limiting expectations of society, and not a place of repression » (47). G. Sand montre donc le couvent comme une possibilité de vie attirante pour les femmes. Avec Rose et Blanche, G. Sand met l'accent sur le réseau de soutien féminin comme édificateur et comme source de réalisation du moi dans le domaine féminin. La suite de l'Histoire montrera le changement de position incontestable de l'auteur et l'affirmation de l'existence d'autres moyens de survie et de réussite personnelle. G. Sand représentera différents types de femmes au cours de sa carrière, refusant ainsi l'uniformité d'une catégorie « femme ». Ce refus souligne le mouvement continu, le devenir toujours en progrès de la femme, en parallèle avec l'histoire, la société et la politique. Pour atteindre une société dans laquelle la femme n'aura plus besoin de s'enfermer dans un couvent pour être heureuse, ou du moins, ne pas subir l'hégémonie patriarcale telle qu'elle se présentait en ce début de siècle, G. Sand insistera sur les enjeux thématiques de l'instruction et de 
l'éducation. Rose se tourne vers l'enseignement du chant aux jeunes filles au couvent. Rose et Blanche présente un auteur encore timide quant à ses aspirations et à leur expression. G. Sand ne s'engage pas encore totalement dans ce combat de longue haleine qui sera le sien. Elle met le doigt sur les sujets sensibles, mais a comme solution, pour ses héroïnes, de se tourner vers la femme et de se retirer du monde qui la persécute.

La solution d'un espace typiquement féminin ne la satisfera pas longtemps. Elle montrera que ce n'est pas à la femme de s'enfermer, mais plutôt, que c'est à la société qu'il revient de changer. La peinture qu'elle fait du couvent n'est, à ce titre, pas toujours très flatteuse dans Rose et Blanche. Le couvent en tant qu'espace, en faisant abstraction de toute idéologie dominante, représente essentiellement un abri face aux intempéries de l'extérieur. En revanche, dès que la religion entre en jeu, la perception du couvent change et s'obscurcit. G. Sand critique ouvertement l'Eglise et ses règles. Si le couvent est représenté principalement comme un asile pour ces jeunes protagonistes, la religion qui s'y pratique n'est évidemment pas ce qui attire les jeunes filles. Le roman est intégralement basé sur la dichotomie mettant en opposition l'intérieur et l'extérieur du couvent qui va de pair avec la dichotomie femme/homme. La femme n'a pas de place à prendre en société, sinon celle d'épouse ou de courtisane (le fait d'être actrice est associé à cet état).

Avec Rose et Blanche, G. Sand se positionne d'abord dans la dialectique de Simone de Beauvoir. Les oppositions binaires homme/ femme, sujet/objet, espace social/espace domestique ou privé sont établies et critiquées. Il s'agit ici des premiers pas de G. Sand en ce qui concerne sa position féministe. Cependant, même si elle critique l'attitude de la société patriarcale à l'égard de la femme, elle situe sa critique dans le cadre même de ces dichotomies. Ce genre de critique renforce cette idée de la femme comme étant l'Autre et réinscrit en fin de compte ce discours féministe comme un acte performatif et constitutif d'un cadre où la femme est effectivement l'Autre, c'est-à-dire, dont l'identité est construite en relation uniquement à l'homme. Après Rose et Blanche, G. Sand confirmera sa position féministe et annonciatrice de la critique féministe moderne, et n'hésitera pas à s'engager plus avant dans la brèche déjà ouverte par ses consoeurs des siècles précédents, comme par exemple Christine de Pisan, Marguerite de Navarre ou Madame de Lafayette, pour n'en citer que quelques-unes. Cependant, ses personnages féminins vis-à-vis de l'espace se trouveront en perpétuel mouvement et occuperont à tous les instants, toutes sortes d'espaces. La femme deviendra donc finalement elle-même, l'espace de sa propre subjectivité. 
Notes

1. Dans la biographie concoctée par Mabel Silver sur Jules G. Sandeau, la description de lieux et de personnages dans Rose et Blanche serait attribuée à George Sand: « Dans les premières lignes, la vieille sœur Olympie [...] est sans doute la création d'Aurore, puisque dans une lettre à sa mère, elle avoue avoir pris une religieuse de sa connaissance pour modèle » (34). La description des Pyrénées, que l'on retrouve d'ailleurs dans Ma Sour Jeanne serait encore, selon Silver, à attribuer à George Sand: « Le paysage grandiose des Pyrénées [. . .] Il paraît hors de doute que c'est Aurore qui les a écrites (les pages), puisqu'en 1825, elle avait fait aux Pyrénées un voyage qui l'avait fortement émue » (34). Et encore: "L'histoire de Rose et Blanche se déroule ensuite dans les environs de Nérac, dans les Landes et à Bordeaux, régions bien connues d'Aurore » (34). G. Sand avouera par la suite « qu'elle avait ébauché ce roman, mais qu'ensuite Jules G. Sandeau le refit en entier » (35).

2. Comme il est souligné dans Women's Writing in 19th Century France: « G. Sand follows previous nineteenth century women's novels in contrasting a sexist society with a primitive Nature that offers a refuge for the beleaguered woman, as in Indiana » (89). Non seulement la dichotomie nature/culture est pertinente dans cette étude sur Rose et Blanche, mais la référence à Indiana et à des dichotomies du même ordre se retrouvera par la suite, dans un des chapitres suivants.

\section{Bibliographie}

Sources primaires :

Sand, George. La Comtesse de Rudolstadt. 1843. Paris: Phébus, 1999.

-Consuelo. 1842. Paris: Phébus, 1999.

Sand, J. Rose et Blanche. 1831. Lavardac: Amis du Vieux Nérac, 1993.

Sources secondaires :

Albistur, Maïté, and Daniel Armogathe. Histoire du féminisme français. Paris:

Des Femmes, 1977.

Aron, Jean-Paul. Misérable et glorieuse: La femme au dix-neuvième siècle.

Paris: Arthème Fayard, 1980.

Bachelard, Gaston. La poétique de l'espace. Paris: PUF, 1981.

Beauvoir, Simone. Le deuxième sexe. Paris: Gallimard, 1949.

Beizer, Janet. Ventriloquized Bodies. Ithaca, NY: Cornell University Press,

1994. 